\title{
HISTORIC GARDENS AND CLIMATE CHANGE
}

\author{
Insights, Desiderata and Recommendations ${ }^{1}$
}

\section{Preamble}

Historic gardens, parks and cultural landscapes are cultural assets worthy of preservation and, like buildings and art collections, irreplaceable testimonies to our civilization. Hence, they are protected, maintained and studied to secure long-term upkeep for society. However, due to climate change which is perceptible worldwide, these gardens and parks are particularly endangered. Between 2016 and 2019, the members of the interdisciplinary research group (IRG) »Historic Gardens and Climate Change« of the Berlin-Brandenburg Academy of Sciences and Humanities devoted themselves to the question of whether and how precautions can be taken for their preservation. Historic gardens display the effects of climate change in a condensed form within a limited area reflecting the resulting consequences for culture, nature and society in equal measures. They are, therefore, particularly suitable for a comparative analysis of options for adaptation to changing climatic conditions.

While historic gardens have been the subject of monument preservation and (garden) art history for many years, natural and social scientists have now, for the first time, jointly researched four gardens as case studies within the IRG. In this way, the perspective of monument conservation and art history was systematically expanded to include a natural, biogeophysical view on historic gardens and an investigation of their role as places of social action. This interdisciplinary perspective was not only a tremendous gain for the participating scientists themselves, but also led to a considerable acquisition of knowledge regarding the development of possible strategies for the preservation of historic gardens under the conditions of climate change. In the course of the discussions it became clear to everyone involved in the IRG that only an interdisciplinary approach is suitable for the longterm preservation of gardens as cultural assets worthy of protection.

This text summarises the IRG's research and outlines initial recommendations which, on the one hand, are addressed to those responsible for the gardens and, on the other hand,

1 This chapter is the result of several panel discussions held by the members of the IRG »Historic Gardens and Climate Change«. 
contain suggestions for politicians and society so as to raise awareness with respect to the functions and values of historic gardens, parks and cultural landscapes.

The starting point for the following considerations is the assumption that the requirements for garden monument conservation have shifted and expanded in view of climate change. The value of historic gardens as testimonies of art and history forms the basis of the conservation mandate. The uniqueness of these gardens lies in the fact that they are, at the same time, immovable natural spaces which, to date, have only minimally been systematically and comprehensively described and recorded from a natural sciences point of view. As social spaces of the past and present, gardens are also a social projection surface and, therefore, of interest to the social sciences. A concluding outlook opens up perspectives on future areas of responsibility for sciences, humanities and politics.

\section{Why is climate change of particular relevance for historic gardens?}

Historic gardens, parks and cultural landscapes take a special place in the canon of arts, as alongside a variety of artistic objects they consist mainly of living materials, especially trees, shrubs, flowers, lawns, meadows, soil and water. Due to the environmental dependency of these artistically arranged materials, the current climate change with its effects (storms, heavy precipitation, heat waves, temperature rise, changed soil conditions, etc.) affects these works of art largely unprotected. As cultural testimonies and a valuable component of human civilization, however, it is crucial to preserve historic gardens on behalf of society.

The outstanding peculiarity of historic gardens lies in the fact that they are spaces and, thus, also systems of culture, nature and society. This means that the climatic changes of the past and the present are reflected in them as if bundled in a magnifying glass. Their structurally rich composition is, on the one hand, an inherent part of (overall) works of art and, on the other hand, it creates diverse natural spaces with high biodiversity, including genetic variety. In addition, historic gardens are mirrors of past societies as well as a place of recreation and education for today's visitors. This functional diversity of historic gardens is threatened in its entirety by the current climate change.

In view of this development, it is the task of sciences, humanities and garden conservation to increasingly communicate and reflect on classical monument criteria and functions (e.g. artistic, historical, scientific, economic, urban planning) and to sound out how social identification potential can be developed from them. Climate change and its impact on historic gardens could play an important role in placing the value of gardens in a new context for the foundation of cultural identities.

\section{Requirements for the preservation of garden monuments}

Historic garden conservation is responsible for maintaining the condition and appearance of gardens on behalf of society. Knowledge and methodology in this respect have been 
developed and handed down in the gardens themselves for about 100 years but have also been taught at universities and technical colleges for about 30 years. Laws on the protection of historic monuments and international agreements consolidate the legal framework and determine the direction of the preservation of historic monuments and gardens. The Florence Charter (1981) is explicitly dedicated to historic gardens, parks and cultural landscapes. It emphasises that the gardens' authenticity rests on the planning of the aesthetic and spatial conception of the various sections, decorative furnishings, plant selection and building materials. A decision on replanting, for example when the previous species can no longer thrive due to climate change, can only be made on the basis of the practical and theoretical knowledge collected on the history of their origin, the individual time layers found in the garden and their respective significance. Historic garden conservation always works in exchange with nature conservation, as not only the artistic, but also the natural features of historic gardens are threatened to be damaged or even irretrievably lost as climate change progresses. As structurally rich landscape elements, historic gardens have developed into refuges of biological diversity, especially in urban and suburban areas. Nevertheless, it is recommended to give priority to the scope of preservation of historic monuments over measures of nature conservation if the desired harmonisation of both objectives cannot be achieved. Compared to other genres of art, the preservation of historic gardens requires a far greater degree of permanent conservation and restoration: Hedges have to be cut, lawns mown, lines of sight kept clear, trees replaced when they reach the age of maturity, etc. In the course of the year, the gardeners must also perform other recurring tasks. The personnel responsible for this broad spectrum of care measures must be appropriately trained and recruited in sufficient numbers, as national and international experts have been urging for some time.

The recommendations for the preservation of historic gardens as a Gesamtkunstwerk are effective at various levels and range from conservation practice to dialogue with relevant social groups and political decision-makers. Against this background, the following recommendations arise from the point of view of preservation of historic monuments:

- The value of the historic gardens is essentially determined by the built infrastructure on their grounds: historic buildings, bridges, paths, artificial and natural waters, walls and statues. These are precisely the elements that are increasingly threatened by climate change. To do justice to the task of preserving historical monuments, it is high time for a regular analysis of the substance of the buildings in historic gardens using cutting-edge technologies. Application of appropriate conservation methods immediately upon first signs of deterioration would be an optimal solution. Thanks to new sensor technologies deeper anomalies can, for example, be concluded from the temperature distribution on the surface of the respective building component part.

- With respect to the role of new technologies - in particular digital technologies - for prevention, risk reduction, monitoring and planning, there is an urgent need to fur- 
ther develop the training profiles in garden monument conservation at all levels. The IRG provides the blueprint for an interdisciplinary structured course of studies. Consideration should also be given to how subordinate vocational training content can be adapted to these new framework conditions. Furthermore, efforts to identify, evaluate and make available handed-down traditional knowledge and experience are urgently required at all levels.

- The sum of use-related and climate-related damage not only results in an increased workload for the gardeners but also in extra organisational efforts. In this respect, measures to increase the number of garden care professionals and to financially strengthen the management sector appear inevitable. Furthermore, an increase in the budget for resources is essential to compensate for losses due to additional damage occurrences.

\section{The testimonial and artistic value of historic gardens}

Historic gardens consist mainly of significant and meaningful spaces and images. In order to be able to determine the measures necessary for their preservation, it is fundamentally necessary to research their individual artistic and monumental value. This evaluation takes place against the background of the discussion on authenticity and originality in garden design, which differs from other genres due to the constant changes in its natural components. Specific benchmarks should be applied, in particular regarding the leeway between conservation and restoration.

Gardens as accessible works of art always have an artistically designed topography that can be described vividly and is related to man and his own movement (kinaesthesia). This is achieved, in particular, through terrain modelling, stretches of water and vegetation arrangements. Buildings, statues, grottos and inscriptions are made accessible via pathways and, thus, draw a programmatic picture and context of meaning (iconology).

However, if, for example, trees that are essential for a garden or one of the garden images gradually die or fall, the artistic value defined by authenticity and originality is lost. This severely reduces the cultural significance and educational value of the respective garden monument.

By classifying and evaluating the formal design in the context of the history and theory of garden art, the significance of the respective gardens can be determined individually, which has already been largely achieved for outstanding gardens (e.g. UNESCO World Heritage List, park maintenance works (Parkpflegewerke)). In view of the increased damage and losses caused by climate change that have already occurred or are becoming apparent, this task is becoming increasingly urgent. It is essential to convince the public and politicians of the important cultural significance of such gardens in order to be able to justify and initiate measures for the preservation of garden monuments. 
Based on the methodological canon of garden art history, original design phases can be reconstructed, i.e. based on source material such as inventory plans, writings and archival documents, but also by means of ground-archaeological investigations. An analysis, for example, of the stock of plantations, water systems, paths, the handling of the topography and architectural features allows scientific statements to be made on the intended artistic effects, which are characteristic for certain regions, cultures, epochs, etc.

The determination of these artistic and historical testimonial values must, in principle, precede all measures, as:

- Decisions regarding the elements that must be preserved or restored in the respective historic garden, park or cultural landscape are based on the expertise in the history of garden art and the preservation of historic monuments.

- In view of the large number of registered garden monuments in Germany, it is strongly recommended that such a description and evaluation should generally precede all practical gardening measures.

- However, catalogues of measures for the preservation of garden monuments can only be drawn up taking into account the scope for action in the natural environment which has to be determined by the natural sciences (see below).

- Information on how to deal with climate and weather in the past must be systematically developed and evaluated in a source-critical way and made available to the natural sciences.

- Based on the evaluation of historical documents from the double perspective of the humanities and natural sciences, the discussion on the scope of adaptation measures in the light of climate scenarios must be redefined.

- In the future, the entire spectrum of expertise drawn in historic gardens since their creation as well as the experience of gardeners should be more closely combined.

\section{Historic gardens as specific natural spaces}

Historic gardens, as habitats equipped with native and foreign species of woody plants, constitute a separate usage category whose functionality, dynamics and beneficial effects have to date been subject to very little scientific research in contrast to other cultural landscapes. Only since the 1980s have historic gardens increasingly become the subject of scientific research. Initially, this was limited mainly to the analysis of the diversity of plant and animal life. With the development of ecosystem research approaches, it has become increasingly clear that near-natural systems are characterised by a complex structure of diverse biotic and abiotic factors (soil, water, vegetation, fauna, climate, humans) that follow general laws but that can vary considerably depending on the respective location. The 
ongoing climate change is currently having a profound impact on the structure of these ecological complexes. Suitable measures for the protection of historic gardens can, therefore, only succeed if they take the respective site conditions into account. For this reason, the lack of information on the specific site characteristics poses new challenges for the conservation of historic monuments, especially in view of climate change. Only a comprehensive knowledge of the natural conditions enables a better assessment of their susceptibility to climatic stress.

Weather and climate have long been the subject of research. Current prediction models forecast a significant increase in long periods of drought, extreme summer temperatures as well as rising temperatures and changing precipitation conditions. However, models for a small-scale differentiated assertion on the effect of the vegetation structure on the local climate are still lacking. Based on case studies, the IRG was able to show that the vegetation structure has an important influence on the microclimate and, thus, on people's well-being as well as biological diversity. Furthermore, it was proven that gardens have a positive influence on the local climate beyond their borders. Corresponding model calculations provide a basis for the modification of the existing structure in order to optimise the micro and local climatic effects of the gardens.

From this, the following recommendations can be derived for the protection of the natural functions of historic gardens against the consequences of climate change:

- As a rule, there are no comprehensive surveys on the substrate properties - especially in deeper soil layers - and on the heterogeneity of the substrates for historic gardens. Therefore, systematic soil mapping is recommended for all historic gardens in order to be able to assess the site potentials in a differentiated manner and to carry out the resulting conservation measures site-specifically. In addition, material flow management should be implemented in order to assess nutrient depletion and humus development in a spatially differentiated manner and to be able to take compensatory measures in line with demand. This can be achieved by internal recycling, but also by fertilisation measures. Aiming at increasing the humus content, mulch concepts should be consistently implemented and soil substrates in tree plantations should be enriched with stable organic matter in order to increase the water and nutrient retention capacity in the root area. Soil improvement measures should be supplemented by targeted periodic nutritional analyses.

- The preservation of genetic diversity forms the basis for the adaptation of vegetation to climate change. Newer genetic analysis methods provide an insight into the respective original region of origin and, thus, provide an indication of the potential resilience of the plant material. The application of appropriate analyses can, therefore, support future decisions on the selection of seeds and planting stock. This applies, in particular, to the characterisation of seeds and planting stock from our own cultivation. It is, therefore, recommended that appropriate genetic analyses be used in parks' own nurseries to assess the climatic adaptability of the plant material. 
- Analogous to plant diversity, the spread of animal species can pose an increasing problem for the maintenance of gardens. The immigration of new animal species (neozoa) is essentially caused by climate change, but the internationalisation of trade and the increase in tourism also play a role. With the help of models, the general distribution dynamics can be estimated very well. Monitoring the regional or local nature of this phenomenon could, therefore, support the solution of such problems in an important area.

- The protection of historic gardens from the consequences of climate change is not possible without foresighted conservation management (precautionary principle). Novel sensor technology and the associated technologies of digitisation offer a wide range of options to record relevant condition data in a timely and georeferenced manner and, thus, to be able to make decisions on monument conservation in a temporally and spatially differentiated manner. Particularly regarding extreme events, the use of innovative technologies can make a major contribution to avoiding or limiting adverse effects.

- A central recommendation of the IRG is, therefore, to use digitisation and modern sensor technology (e.g. soil water content, carbon content, nutrient content) and visualisation instruments in order to be able to carry out and simultaneously document management measures in a timely and needs-based manner. It would be desirable to supplement such a system with historical data and to further develop it into a garden archive in the sense of historic garden conservation and art history. In the long term, this could lead to the development of approaches for artificial intelligence which, based on sensory networks, would enable the development of self-learning systems for prevention and protection against risks.

\section{Historic gardens as an object of social sciences}

Historic gardens, parks and cultural landscapes as works of art and exceptional natural spaces are ultimately always embedded in a social context as they are still today being used, valued and administered by humankind. Therefore, their social anchoring together with highly varied demands for use also has an influence on the resilience of historic gardens to climate change. It is, thus, advisable for those responsible for such gardens to acknowledge the wishes and needs of the various user groups (including tourists and residents) and to proactively involve these groups in decisions on preservation measures.

At the same time, those responsible for historic gardens are constantly faced with the task of developing strategies for mobilising economic resources, social legitimisation, political assertiveness or dealing with local »Friends and Supporters «. According to the IRG, however, perspectives on climate change are far from clear, as the consequences of climate change are assessed in different ways and sometimes even considered to be a negligi- 
ble issue. Thus, in addition to opportunities, limitations also arise in dealing with social framework conditions related to historic gardens. Sociological studies on the organisational environment should, therefore, be specifically tailored to each individual garden.

Future research on historic gardens should more visibly incorporate social science expertise as a third pillar alongside cultural and natural sciences in order to gain a holistic picture. Only a communication strategy that is well adapted to the respective target groups and that is based on a comprehensive overview of knowledge will make it possible for politicians and society to take greater responsibility in the future and to create framework conditions under which historic gardens can continue to create identity, promote health and provide a wide range of education.

In view of climate change, it is necessary from a social science perspective to preserve historic gardens as follows:

- Importance should be attached to further improving the existing governance structures and to reviewing their suitability, in particular, in order to do justice to the manifold embeddings of gardens in their social and political environment. Without suitable structures - such as the absence of a garden administration - it is not possible to maintain garden monuments, nor is it possible to influence the behaviour of visitors.

- The potential of sociological framing and economic upgrading of historic gardens could be better exploited in the future by reflecting on experiences with the concept of ecosystem services from nature conservation.

- Concrete case studies by the IRG provide initial indications of how changed societal demands and the demand for protection resulting from climate scenarios can be reconciled with the preservation of historic monuments: A shift in the perception of historic gardens as places of leisure to an emphasis on their monumental status can lead to a new potential for increased (idealistic and financial) support and a new appreciation by the public.

- In order to avoid conflicts of use, which exist in many places between recreational demands and the status of the garden as a listed cultural asset, it is essential to control visitor behaviour by means of clearly formulated and comprehensible park regulations as well as further behaviour-shaping measures.

- Historic gardens are ideal places to develop narratives that seize climate change as both an aspect of history and an experience of the present. Corresponding educational offers can contribute to a change in the understanding of nature and to a better knowledge of the value and significance of historic gardens. Such offers can also be used to communicate the effort involved in maintaining such gardens.

- A further recommendation is, therefore, to increase public awareness of the diversity of the beneficial effects emanating from these gardens. At the same time, open spaces for recreation should be created in the gardens and planned in line with easing vulnerable areas. 
- Ultimately, the gardens as archives offer considerable potential for knowledge. It is the continuous contact, in particular, with young people that seems appropriate here to establish a sustainable and effective basis for the appreciation of historic gardens. For example, experiences from the world of theatre could be integrated with suitable formats in the future. A higher presence of technical staff should also increase the inhibition threshold against misuse. Above all, however, such personnel can fulfil an important function in imparting knowledge and, thus, make a decisive contribution to the appreciation of the gardens. In this respect, the gardens should be given a much more comprehensive educational mandate and, in this way, do even more justice to their function as points of socio-historical identification.

- The above recommendations can, generally speaking, only be implemented with additional investment. The willingness to invest appropriate financial funds can only arise if there is an appropriate appreciation for historic gardens in society and politics.

\section{Concluding remarks and outlook}

In conclusion, it must be kept in mind that the complex inter- and transdisciplinary interwoven processes that have influenced the individual historic garden since its creation should always be taken into account. Only on the basis of this broadly-defined knowledge can we succeed in maintenance or adaptation to climate change.

Considering the approximately 35,000 garden monuments in Germany, comprehensive natural and social science expertise is required in research and education as a second and third pillar of knowledge, in addition to art historic fundamentals (park maintenance works). The diverse, sometimes new needs and requirements that historic gardens encounter in the face of climate change could be met by this bundled expertise.

In view of climate change, it is a must to understand historic gardens as cultural monuments even more comprehensively as objects of research. Without scientific backing and constant research on the above-mentioned topics, respective sustainable conservation, adaptation or restoration measures cannot possibly be designed by the garden preservation authorities. In this comprehensive sense, science-based historic garden conservation can be exemplary for other areas of cultural heritage. A journalistic reappraisal and documentation of the listed cultural monuments is well advised to begin here. Furthermore, it should impart, in effective public communication, general basis knowledge and the manifold implications and functions of garden art, thus, allowing for today's society to better identify with and interpret historic gardens on the whole. 